\title{
Performance Evaluation of Energy Detector for Cognitive Radio Network
}

\author{
Rubeena Sethi (M.Tech Student) ${ }^{1}$, Indu Bala (Associate Professor) ${ }^{2}$ \\ ${ }^{I}$ (Electronics \& Communication Engineering Department Chandigarh Engineering College/ Punjab Technical \\ University, India) \\ ${ }^{2}$ (Electronics \& Communication Engineering Department, Chandigarh Engineering College/ Punjab Technical \\ University, India)
}

\begin{abstract}
Cognitive Radio is an intelligent radio that exploit electromagnetic spectrum opportunistically by using different techniques. The critical task is to sense the free spectrum and using it. In past few years many sensing schemes have been proposed to sense the availability of free spectrum. In this paper, Energy Detector technique of spectrum sensing is reviewed. The results have been simulated for Probability of False Alarm, Probability of Detection and Probability of Missing detection for the Energy Detector.
\end{abstract}

Keywords : Analog to Digital converter(A/D), Cognitive Radio, Defense Advanced Research Projects Agency (DARPA), Energy Detector, NeXt Generation $(x G)$, Radio Frequency (RF),Signal to Noise Ratio(SNR), Spectrum Sensing.

\section{Introduction}

Due to fixed spectrum assignment policy of channels, utilization of the spectrum band got limited for today's wireless networks. Hence new paradigm is necessitated for the efficient use of the spectrum. DARPA proposed a new approach for Dynamic Spectrum Access Network, called Next generation. The key technology of the $\mathrm{xG}$ is Cognitive Radio. Cognitive Radio is an intelligent radio that exploits electromagnetic spectrum opportunistically by using different techniques. The critical task is to sense the free spectrum and using it [4]. Main functions for cognitive radios in $\mathrm{xG}$ networks can be summarized as follows:

- Spectrum sensing: Determine which portions of the spectrum are available and detect the presence of licensed users when a user operates in a licensed band.

- Spectrum management: Capturing the best available spectrum to meet user communication requirements.

- Spectrum mobility: Maintaining seamless communication requirements during the transition to better spectrum and coordinate with other users.

-Spectrum sharing: Providing the fair spectrum scheduling method i.e. vacate the channel when a licensed user is detected.

\section{Cognitive Radio}

Cognitive radio is an intelligent radio technology in which the radio can change its transmitter parameters according to the environmental conditions. In this technology primary users have higher priority than secondary users. Cognitive radio capabilities are provided to the secondary users. Two main characteristics of the cognitive radio can be defined [1,3]:

Cognitive capability: Cognitive capability refers to the ability of the radio technology to capture or sense the information from its radio environment by the sophisticated techniques in such a way that it should avoid interference to other users to identify the unused portion of spectrum at specific time or location. The steps of the cognitive cycle as shown in Figure1 are as follows:

-Spectrum sensing: It monitors the available spectrum bands, captures their information, and then detects the spectrum holes.

-Spectrum analysis: The characteristics of the spectrum holes that are detected through spectrum sensing are estimated.

-Spectrum decision: The appropriate spectrum band is chosen according to the spectrum characteristics and user requirements. So that communication can be performed over this spectrum band. 


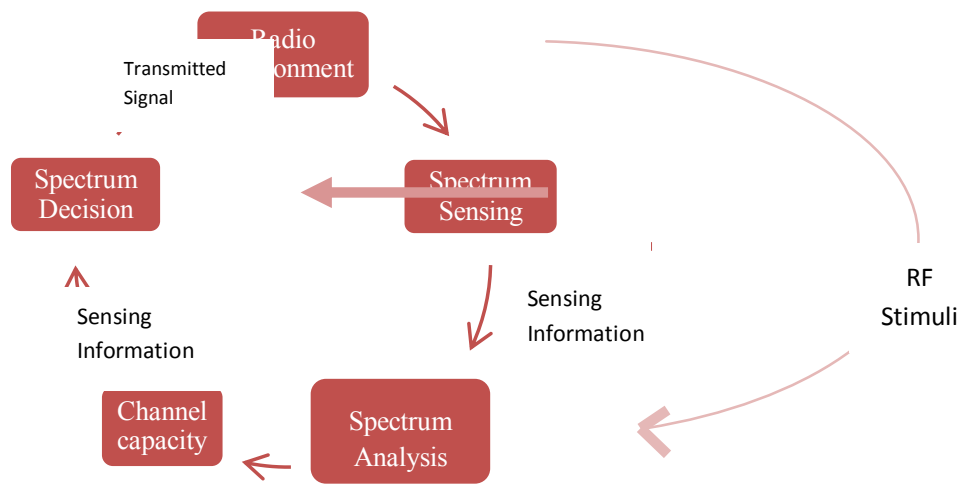

Fig. 1 Cognitive Cycle

Reconfigurability: To obtain the best available spectrum the radio is to be dynamically programmed to transmit and receive on a variety of frequencies. The most important challenge is to share the licensed spectrum without interfering with the transmission of other licensed users as illustrated in Figure2. The cognitive radio enables the usage of temporally unused spectrum, which is referred to as spectrum hole or white space [1].

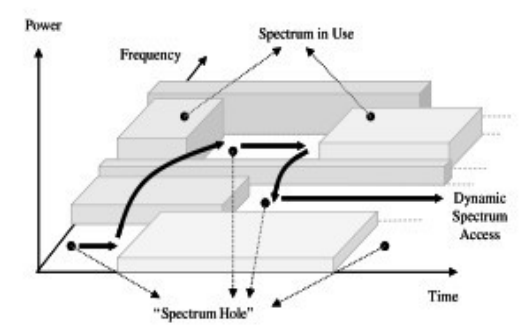

Fig. 2 Spectrum hole concept

If this band is further used by a licensed user, the cognitive radio moves to another spectrum hole or stays in the same band to avoid interference as shown in Figure3. There are several reconfigurable parameters that can be incorporated into the cognitive radio [2] are Operating frequency, Modulation, Transmission power, Communication technology.

\section{Physical architecture of the cognitive radio}

A physical architecture of a cognitive radio transceiver is shown in Figure 3. The main components of a cognitive radio transceiver are the radio front-end and the baseband processing unit and can be reconfigured via a control bus to adapt to the time-varying RF environment. In the RF front-end, the received signal is amplified, mixed and A/D converted. In the baseband processing unit, the signal is modulated/demodulated and encoded/decoded.
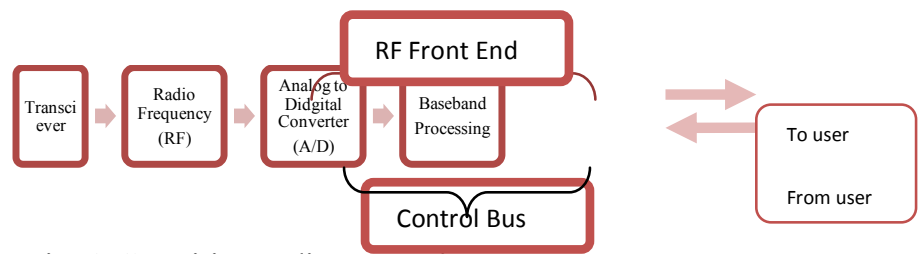

Fig. 3 Cognitive radio transceiver

The novel characteristic of cognitive radio transceiver is a wideband sensing capability of the RF front-end. This function is mainly related to RF hardware technologies such as wideband antenna, power amplifier, and adaptive filter. RF hardware for the cognitive radio should be capable of tuning to any part of a large range of frequency spectrum.

\section{Spectrum Sensing}

The spectrum sensing function enables the cognitive radio to adapt to its environment by detecting spectrum holes. As the present literature for the spectrum sensing is still in its early stages of development [5]. So many challenges are associated with spectrum sensing and further work is in progress to overcome these challenges. Different methods are proposed for identifying the presence of signal transmissions. Some approaches for the spectrum sensing are given in Table I. 
TABLE I. COMARISON OF DIFFERENT SPECTRUM SENSING TECHNIQUES

\begin{tabular}{|l|l|l|l|l|}
\hline Spectrum Sensing & $\begin{array}{l}\text { Prior } \\
\text { Information } \\
\text { of primary } \\
\text { users }\end{array}$ & $\begin{array}{l}\text { Performance in } \\
\text { SNR }\end{array}$ & $\begin{array}{l}\text { Implime-- } \\
\text { ntation }\end{array}$ & $\begin{array}{l}\text { Computational } \\
\text { Cost }\end{array}$ \\
\hline $\begin{array}{l}\text { Matched Filter } \\
\text { Detection }\end{array}$ & Required & $\begin{array}{l}\text { Can work in low } \\
\text { SNR }\end{array}$ & Complex & Low \\
\hline Energy Detection & Not Required & $\begin{array}{l}\text { Can't work in low } \\
\text { SNR }\end{array}$ & Simple & Low \\
\hline $\begin{array}{l}\text { Cyclostationary } \\
\text { Feature Detection }\end{array}$ & Required & Robust in low SNR & Complex & High \\
\hline $\begin{array}{l}\text { Waveform Based } \\
\text { Sensing }\end{array}$ & Required & Good performance & Complex & High \\
\hline $\begin{array}{l}\text { Cooperative } \\
\text { Detection }\end{array}$ & Required & $\begin{array}{l}\text { Can work in low } \\
\text { SNR }\end{array}$ & Complex & High \\
\hline $\begin{array}{l}\text { Interference Based } \\
\text { Detection }\end{array}$ & Required & $\begin{array}{l}\text { Can't work in low } \\
\text { SNR }\end{array}$ & Complex & High \\
\hline
\end{tabular}

In this paper, Energy Detector Based Sensing for the cognitive radio is explained.

\section{Energy Detector Based Sensing}

Energy detector based approach, also known as radiometry or periodogram, is the most common way of spectrum sensing because of its low computational and implementation complexities. In addition, it is very generic as receivers do not need any knowledge on the primary users' signal. The signal is detected by comparing the output of the energy detector with a threshold which depends on the noise floor .Some of the challenges with energy detector based sensing include selection of the threshold for detecting primary users, inability to differentiate interference from primary users and noise, and poor performance under low signal-tonoise ratio (SNR) values. Moreover, energy detectors do not work efficiently for detecting spread spectrum signals [1], [3].

Let us assume that the received signal has the following simple form

$$
y(n)=s(n)+w(n)
$$

where $\mathrm{s}(\mathrm{n})$ is the signal to be detected, $\mathrm{w}(\mathrm{n})$ is the additive white Gaussian noise (AWGN) sample, and $\mathrm{n}$ is the sample ind Note that $\mathrm{s}(\mathrm{n})=0$ when there is no transmission by primary user.

The decision metric for the energy detector can be written as

$$
M=\sum_{n=0}^{N}[|y(n)|]^{2}
$$

Where $\mathrm{N}$ is the size of the observation vector. The decision on the occupancy of a band can be obtained by comparing the decision metric $\mathrm{M}$ against a fixed threshold $\lambda_{\mathrm{E}}$. This is equivalent to distinguishing between the following two hypotheses:

$$
\begin{aligned}
& \mathcal{H}_{0}: y(n)=w(n) \\
& \mathcal{H}_{1}: y(n)=s(n)+w(n)
\end{aligned}
$$

The performance of the detection algorithm can be summarized with two probabilities: probability of detection $P_{d}$ and probability of false alarm. $P_{d}$ is the probability of detecting a signal on the considered frequency when it truly is present. Thus, a large detection probability is desired. It can be formulated as

$$
P d=\operatorname{Pr}\left(M>\lambda_{E} \mid H 1\right)
$$

$\mathrm{P}_{\mathrm{f}}$ is the probability that the test incorrectly decides that the considered frequency is occupied when it actually is not, and it can be written as

$$
P f=\operatorname{Pr}\left(M>\lambda_{E} \mid H 1\right)
$$

$\mathrm{P}_{\mathrm{f}}$ should be kept as small as possible in order to prevent underutilization of transmission opportunities. The decision threshold $\left(\lambda_{E}\right)$ can be selected for finding an optimum balance between $P_{d}$ and $P_{f}$. A great challenge of spectrum sensing for the cognitive radio is to detect the presence of the primary transmitter with little information about the channel $\mathrm{h}$ and the transmitted signal $\mathrm{s}(\mathrm{t})$. In such a scenario, the energy detector has been shown as the optimal detector for a zero-mean constellation of $s(t)$ [2]. Specifically, the energy of the received signal, denoted by, is collected in a fixed bandwidth $\mathrm{W}$ and a time slot duration $\mathrm{T}$ and then 
compared with a predesigned threshold $\lambda_{\mathrm{E}}$. If $y>\lambda_{\mathrm{E}}$, then the cognitive radio assumes that the primary system is in operation, i.e. $\mathcal{H}_{1}$. Otherwise, it assumes $\mathcal{H}_{0}$.

The average probability of false alarm, detection and missing of energy detection over Rayleigh fading channels can be given respectively.

$$
\begin{aligned}
\mathrm{P}_{\mathrm{f}} & =\mathrm{E} \gamma\left[\operatorname{Prob}\left\{\mathcal{H}_{1} \mid \mathcal{H}_{0}\right\}\right] \\
& =\frac{\Gamma\left(u, \frac{\lambda}{2}\right)}{\Gamma(u)}
\end{aligned}
$$

$\mathrm{P}_{\mathrm{d}}=\mathrm{E} \gamma\left[\operatorname{Prob}\left\{\mathcal{H}_{1} \mid \mathcal{H}_{1}\right\}\right]$

$=e^{-\frac{\lambda}{2}} \sum_{n=0}^{u-2}\left(\frac{1}{n !}\right)\left(\frac{\lambda}{2}\right)^{2}+\left[\frac{(1+S N R)}{S N R}\right]^{u-1} *\left[e^{-\frac{\lambda}{2(1+S N R)}}-e^{-\frac{\lambda}{2}} \sum_{n=0}^{u-2}\left(\frac{1}{n !}\right)\left[\frac{\lambda * S N R}{2(1+S N R)}\right]^{n}\right]$

$\mathrm{P}_{\mathrm{m}}=\mathrm{E} \gamma\left[\operatorname{Prob}\left\{\mathcal{H}_{0} \mid \mathcal{H}_{1}\right\}\right]$

$$
=1-P_{\mathrm{d}}
$$

where SNR is average SNR at the cognitive radio.

$\mathrm{E} \gamma[\cdot]$ represents the expectation over the random variable $\gamma$ which is Rayleigh distributed.

$\operatorname{Prob}\{\cdot\}$ stands for the probability.

$\Gamma(\cdot, \cdot)$ is the incomplete gamma function and

$\Gamma(\cdot)$ is the gamma function.

Finally, $\mathrm{u}=\mathrm{TW}$ with $\mathrm{u}=5$ is used throughout this paper.

In order to measure the energy of the received signal, the output signal of bandpass filter with bandwidth $\mathrm{W}$ is squared and integrated over the observation interval T. Finally, the output of the integrator, $\mathrm{Y}$, is compared with a threshold, $\lambda$ to decide whether a licensed user is present or not [1], the probability of detection $\left(\mathrm{P}_{\mathrm{d}}\right)$ and probability false alarm $\left(\mathrm{P}_{\mathrm{f}}\right)$ are given as follows:

$$
\begin{gathered}
P d=P\left\{Y>\lambda / \mathcal{H}_{1}\right\}=Q m(\sqrt{2 \gamma}, \sqrt{\lambda}) \\
P f=P\left\{Y>\lambda / \mathcal{H}_{0}\right\}=\Gamma(m, \lambda / 2) / \Gamma(m)
\end{gathered}
$$

Where $\gamma$ is the SNR, $\mathrm{u}=\mathrm{TW}$ is the time bandwidth product, $\Gamma($.$) and \Gamma(.,$.$) are complete and incomplete$ gamma functions and $\mathrm{Q}_{\mathrm{m}}(\mathrm{)}$ ) is the generalized Marcum Q-function. From the above functions, while a low $P_{d}$ would result in missing the presence of the primary user with high probability which in turn increases the interference to the primary user, a high $\mathrm{P}_{\mathrm{f}}$ would result in low spectrum utilization since false alarms increase the number of missed opportunities. Since it is easy to implement, the recent work on detection of the primary user has generally adopted the energy detector $[2,3]$.

\section{Results}

As shown in Figure4, probability to detect the false alarm decreases as the value of $u$ increased. Value of threshold is taken as 1 for this result.

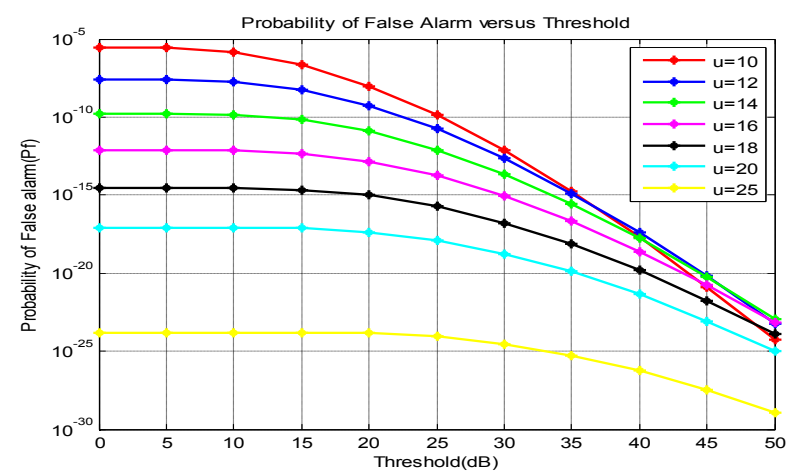

Fig. 4 Probability of False alarm versus Threshold

Figure5 and Figure 6 are shown for probability of detection for the smaller values of threshold, and for the higher values of threshold by keeping time bandwidth product 5 respectively. Here in Figure5 Probability of detection vs. SNR is shown. It is analyzed that as the value of threshold set high, it lowers the probability of detection. So to get the optimize value of probability of detection the value of threshold should be set as small as possible. In this graph it can be clearly analyzed that probability of detection is maximum when value of lameda is smallest. In this paper two graphs are taken to show the probability of detection at different values of probability of false alarm. 


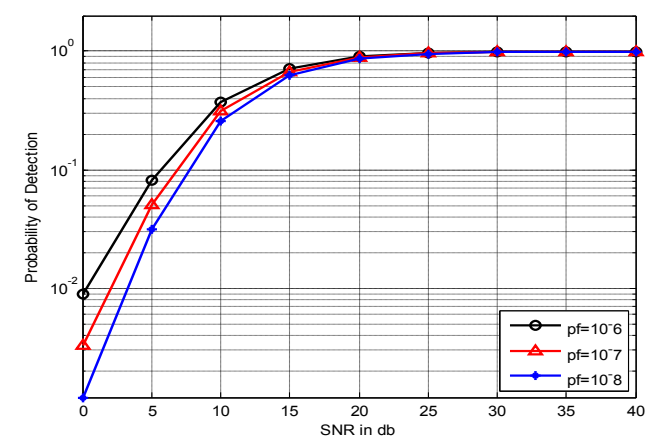

Fig. 5 Probability of detection versus SNR

Figure 6 shows how probability of detection varies for the higher values of probability of false alarm. It can be seen that increasing value of threshold, the probability of detection decreases and after $15 \mathrm{db}$ of SNR probability of detection shows no variation.

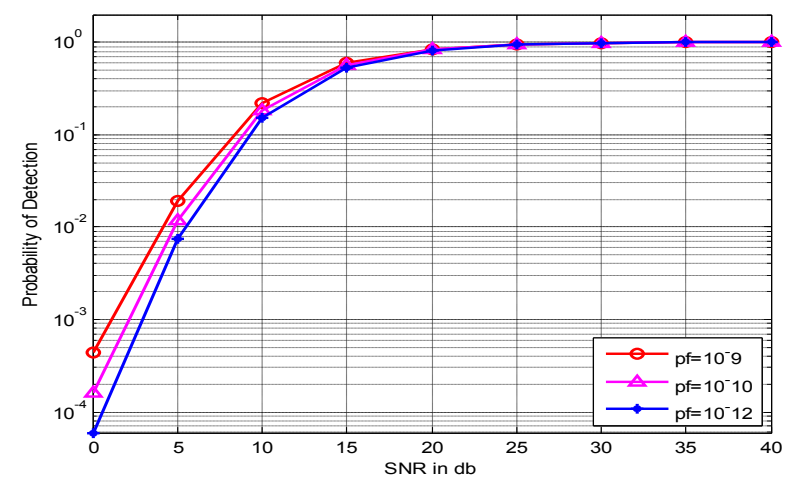

Fig. 6 Probability of detection versus SNR

As seen in Figure7, probability of missing is exactly the reciprocal of probability of detection, as shown in Figure5 and Figure6. It could be seen that as the value of probability of false alarm increases there is increase in the probability of miss detection.

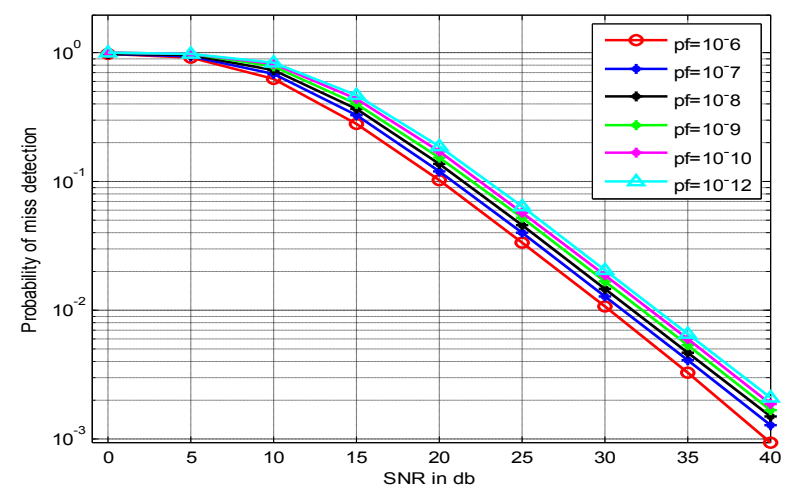

Fig. 7 Probability of miss detection versus SNR

\section{Conclusion}

In this paper performance evaluation of energy detector for spectrum sensing in cognitive radio is done and results have been simulated in MATLAB 7.1 for probability of false alarm and probability of miss detection.

It has been observed that the probability of detection can be improved for given time bandwidth product by keeping threshold value small. The results for probability of detection and probability of miss detection have been simulated for different values of SNR. It has been observed that probability of detection improves as SNR increases beyond $15 \mathrm{~dB}$ and probability of miss detection is decreasing. 


\section{Future Scope}

The new interpretation of spectrum space creates new opportunities and challenges for spectrum sensing while solving some of the traditional problems several sensing methods are there like Cyclostationary, Matched Filtering, Waveform based sensing, and Radio identification based sensing. Pro-active approaches and sensing methods can be employed in current wireless. Estimation of spectrum usage in multiple dimensions including time, frequency, space, angle, and code; identifying opportunities in these dimensions; and developing algorithms for prediction into the future using past information can be considered as some of the open research areas. In this way lot of work and experiment can be done on different parameters of different techniques to get the better results in spectrum sensing for cognitive radios.

\section{References}

[1]. Yucek and Arslan, “A survey of spectrum sensing algorithms for cognitive radio applications" IEEE communications surveys \& tutorials vol.11, No.1,pp 116-130, first quarter 2009.

[2]. Karama Hamdi, Wei Zhang, and Khaled Ben Letaief, "Power control in cognitive radio systems based on spectrum sensing side information", IEEE communications society, pp 5161-5165, proceedings ICC 2007

[3]. Ian F. Akyildiz, Won-Yeol Lee, Mehmet C. Vuran, Shantidev Mohanty "Next generation/dynamic spectrum access/cognitive radio wireless networks :A survey", Computer Networks 50 (2006) 2127-2159.

[4]. Federal Communications Commission, "Notice of proposed rulemaking: Unlicensed operation in the TV broadcast bands," ET DocketNo.04-186(FCC04-113), May2004.

[5] S.Haykin, "Cognitive radio: brain- empowered wireless communications,"IEEE Transactions on Communications, vol.23no.2,pp.201-220,2005. 